\title{
Is there more than meets the eye? Presence and role of sub-micron grains in debris discs
}

\author{
P. Thebault and Q. Kral \\ LESIA - Observatoire de Paris, UPMC Université Paris 06, Université Paris-Diderot, Paris, France \\ e-mail: philippe.thebault@obspm.fr
}

Received 22 February 2019 / Accepted 10 April 2019

\begin{abstract}
Context. The presence of sub-micron grains has been inferred in several debris discs, usually because of a blue colour of the spectrum in scattered light or a pronounced silicate band around $10 \mu \mathrm{m}$, even though these particles should be blown out by stellar radiation pressure on very short timescales. So far, no fully satisfying explanation has been found for this apparent paradox.

Aims. We investigate the possibility that the observed abundances of sub-micron grains could be naturally produced in bright debris discs, where the high collisional activity produces them at a rate high enough to partially compensate for their rapid removal. We also investigate to what extent this potential presence of small grains can affect our understanding of some debris disc characteristics.

Methods. We used a numerical collisional code to follow the collisional evolution of a debris disc down to sub-micron grains far below the limiting blow-out size $s_{\text {blow }}$. We considered compact astrosilicates and explored different configurations: A and G stars, cold and warm discs, bright and very bright systems. We then produced synthetic spectra and spectral energy distributions, where we identified and quantified the signature of unbound sub-micron grains.

Results. We find that in bright discs (fractional luminosity $\gtrsim 10^{-3}$ ) around A stars, the number of sub-micron grains is always high enough to leave detectable signatures in scattered light where the disc colour becomes blue, and also in the mid-IR ( $10 \lesssim \lambda \lesssim 20 \mu \mathrm{m})$, where they boost the disc luminosity by at least a factor of 2 and induce a pronounced silicate solid-state band around $10 \mu \mathrm{m}$. We also show that with this additional contribution of sub-micron grains, the spectral energy distribution can mimic that of two debris belts separated by a factor of $\sim 2$ in radial distance. For G stars, the effect of $s \leq s_{\text {blow }}$ grains remains limited in the spectra although they dominate the geometrical cross section of the system. We also find that for all considered cases, the halo of small (bound and unbound) grains that extends far beyond the main disc contributes to $\sim 50 \%$ of the flux up to $\lambda \sim 50 \mu \mathrm{m}$ wavelengths.
\end{abstract}

Key words. planets and satellites: formation - circumstellar matter

\section{Introduction}

An important fraction of main-sequence stars are known to be surrounded by debris discs, which are detected by the luminosity excess that is produced by small dust particles. In the classical view of these systems, this dust is interpreted as being produced by the steady collisional grinding of material that is left over from the planet formation process. The dust-producing collisional cascade is believed to extend from planetesimal-sized parent bodies down to grains that are small enough to be blown out by stellar radiation pressure (Wyatt 2008). For a canonical collisional equilibrium size-distribution in $\mathrm{d} N \propto s^{-3.5} \mathrm{~d} s$, the geometrical cross section of the system is dominated by the smallest grains, of size $s_{\min }$, in the cascade. These smallest grains should thus also dominate the disc luminosity at all wavelengths shorter than $\sim 2 \pi s_{\min }{ }^{1}$, that is, typically from the visible up to the near- to mid-IR. At these wavelengths, observations are thus expected to be mostly sensitive to grains of sizes $s_{\text {min }} \sim s_{\text {blow }}$, where $s_{\text {blow }}$ is the limiting size below which particles are blown out by stellar radiation pressure. Even though detailed numerical collisional models have shown that a realistic grain size distribution can significantly depart from the idealized $\mathrm{d} N \propto s^{-3.5} \mathrm{~d} s$ power law, they all confirmed that the geometrical cross section is probably still dominated by grains close to the $s_{\text {blow }}$ value

1 Because for a given wavelength $\lambda$, both the scattering and emission efficiencies quickly drop for sizes smaller than $\lambda / 2 \pi$ (e.g. Li 2008).
(Thebault et al. 2003; Krivov et al. 2006; Thebault \& Augereau 2007).

However, this theoretical prediction is sometimes challenged by observations. For example, the detailed studies by Pawellek et al. (2014) and Pawellek \& Krivov (2015) have shown that for solar-type stars at least, $s_{\min }$ can be significantly larger than $s_{\text {blow }}$, with $s_{\text {min }} / s_{\text {blow }}$ reaching values up to $\sim 10$. Several explanations have been proposed for this puzzling feature, such as the non-production of very small grains resulting from the conservation of the surface energy at collisions (Krijt \& Kama 2014; Thebault 2016) or the imbalance between small grain production and destruction rates in dynamically "cold" discs (Thebault \& Wu 2008; Thebault 2016). For a handful of systems, however, the problem is the opposite: observations have revealed the signature of sub-micron grains that are smaller than the blow-out size. This presence has been inferred either from a blue colour in scatteredlight photometry, such as for HD 32297 (Kalas 2005; Fitzgerald et al. 2007a), HD 15115 (Debes et al. 2008b), or AU Mic discs (Augereau \& Beust 2006; Fitzgerald et al. 2007b; Lomax et al. 2018) or by strong solid-state features in the mid-IR, such as in the HD 172555 (Johnson et al. 2012) or HD 113766 systems (Olofsson et al. 2013) 2 .

2 We leave aside the probably completely different problem of exozodiacal discs for which a large population of sub-micron or even nano grains has been detected extremely close to their parent stars (see review by Kral et al. 2017). 
The presence of significant amounts of $s \leq s_{\text {blow }}$ dust is unexpected because these grains should be blown out of the system on very short timescales, of the order of one orbital period. Given this strong constraint, most explanations proposed for this puzzling presence involve a recent stochastic event, such as the catastrophic breakup of a large planetesimal (Johnson et al. 2012; Olofsson et al. 2013; Kral et al. 2015) or the powerful collisional chain reaction, also triggered by the breakup of a planetesimal, that is called collisional "avalanches" (Grigorieva et al. 2007; Thebault \& Kral 2018). However, these scenarios have the disadvantage of relying on short-lived events that are probably unlikely to be observed.

We here reconsider this question of unbound grains within the context of standard debris discs at collisional steady state, and investigate under which circumstances these discs can "naturally" produce a steady level of small sub-micron grains that could leave a long-lived signature. There are indeed several reasons why small $s \leq s_{\text {blow }}$ grains might, in some cases, be potentially observable in discs at collisional steady-state.

A first potential case is that of bright and dense discs with a very high collisional activity, where we expect the depletion of grains below the $s=s_{\text {blow }}$ limit to be significantly reduced: while the abundances of $s \geq s_{\text {blow }}$ grains, which are produced and destroyed in the collisional cascade, are $\propto M_{\text {disc }}$ ( $M_{\text {disc }}$ being the total disc mass), the abundances of $s \leq s_{\text {blow }}$ grains, which are only produced in the cascade before leaving the system, scale with $M_{\text {disc }}^{2}$. To first order, the density drop at the $s=s_{\text {blow }}$ frontier should thus be $\propto 1 / M_{\text {disc }}$ and should thus decrease for discs with higher collisional activity (i.e. total mass). So far, the amplitude of the density drop at $s=s_{\text {blow }}$, and in particular, its link to a given star or disc configuration, has not been investigated by numerical models studying the collisional evolution of debris discs, even though this drop appears in most of these studies (Thebault et al. 2003; Thebault \& Augereau 2007; Krivov et al. 2006, 2018). To our knowledge, the only study focusing on the $s=s_{\text {blow }}$ frontier has been made by Krivov et al. (2000), which predates the advent of sophisticated collisional models and only considers the specific case of the $\beta$ Pictoris disc.

Another potential reason for the presence of sub-micron grains is that the simplified assumption that the ratio $\beta(s)$ between radiation pressure and gravitational forces is $\propto 1 / s$ and thus continues to increase with decreasing sizes, breaks down below a given size $s_{\text {peak }}$ where $\beta$ reaches its maximum value. For $s \leq s_{\text {peak }}$ grains, $\beta$ even decreases with decreasing sizes, and for solar-type stars and for most standard materials (astrosilicates, carbonaceous grains, etc.), $\beta(s)$ exceeds the limiting $\beta=0.5$ value for blow-out only in a relatively narrow size range around $s_{\text {peak }}$ (see e.g. Krivov et al. 1998). Therefore, very small grains will not be blown out of the system. This explanation has been advocated by Johnson et al. (2012) to explain the presence of sub-micron grains in the HD 172555 system, but only with simple qualitative arguments.

Last but not least, even if they are underabundant, small unbound grains could leave a significant signature in thermal emission because their temperature is generally higher than that of larger grains closer to the black-body value. This effect could be especially pronounced in the Wien domain of the Planck function, where the emitted flux varies exponentially with the temperature.

The present paper is organized as follows. Section 2 presents the numerical model that we use to investigate the collisional evolution of debris discs, in particular, of their size distribution down to sub-micron grains. Section 3 presents our main results for the different star and disc configurations that we have explored. We discuss the implications of these results in light of the observed characteristics of bright debris discs in Sect. 4, and give our main conclusions and perspectives in Sect. 5 .

\section{Model}

We used an improved version of the collisional model developed by Thebault et al. (2003) and Thebault \& Augereau (2007), which is based on a statistical particle-in-a-box approach, where particles are sorted into logarithmic size bins. This code also has a 1D spatial resolution, being divided into radially concentric annuli.

\subsection{Updated procedure for estimating the impact rate}

In versions of the code that were used so far, collision rates and impact velocities were computed using analytical equations based on the average orbital eccentricity and inclinations in each size and spatial bin (or analytical estimates of the outbound velocities for $s_{\text {blow }}$ grains), taking into account the effect of stellar radiation pressure on small grains.

In order to obtain more accurate estimates that for instance take in account that grains are not launched from perfectly circular orbits, we have changed this procedure. We now first run an $\mathrm{N}$-body code, where we deterministically follow the dynamical evolution, taking the effect of stellar gravity and radiation pressure into account, of $\sim 10^{7}$ particles released from an extended disc of virtual parent bodies and covering the complete size range considered in the statistical code. The $N$-body code is an adaptation of the collision-tracking deterministic code developed by Thebault et al. (2006) in the context of mutual planetesimal collisions in binary systems. All mutual close encounters are tracked and relative velocities at encounters are stored in a fivedimension table $\mathrm{d} v\left(r, s_{1}, s_{2}, r_{1}, r_{2}\right)$, where $r$ is the radial location of the encounter, $s_{1}$ and $s_{2}$ are the sizes of the two impacting bodies, and $r_{1}$ and $r_{2}$ the location from which they were released (which can strongly depart from $r$ for grains that are significantly affected by radiation pressure). This huge $\mathrm{d} v\left(r, s_{1}, s_{2}, r_{1}, r_{2}\right)$ table is then reused in the main statistical code ${ }^{3}$.

\subsection{Updated procedure for the collision outcome}

Collision outcomes are divided into two categories, cratering and fragmentation, depending on the ratio between the specific impact kinetic energy and the specific shattering energy $Q^{*}$, which depends on object sizes and composition. As in most similar codes, we consider two distinct prescription for $Q^{*}$ : one for the gravity regime dominating for large $(\geq 0.1-1 \mathrm{~km})$ bodies, and one for the strength regime dominating for smaller objects.

In most collisional codes, the strength regime $Q_{s}^{*}(s)$ is described by a power law with a negative exponent, using prescriptions derived from laboratory experiments (Housen \& Holsapple 1999) or smoothed particle hydrodynamics (SPH) simulations (Benz \& Asphaug 1999). We note, however, that these prescriptions are designed to fit a size regime that typically spans the size domain of $1 \mathrm{~cm}$ to $100 \mathrm{~m}$, and that there is no guaranty that they might be extrapolated over several orders of magnitude into the micron or sub-micron size domain. As

\footnotetext{
3 These estimated velocities in practice never strongly depart from analytical estimates, but this numerical procedure ensures a better accuracy and dispenses with analytical calculations that can become cumbersome for non-circular launch orbits and averaging over all possible encounter angles.
} 
Heng \& Tremaine (2010) correctly pointed out, the prescription has to break down at some point because it would otherwise diverge to infinity for infinitely small bodies. Heng \& Tremaine (2010) and van Lieshout et al. (2014) argued that the experimental results of Flynn \& Durda (2004) showed that $Q_{s}^{*}(s)$ levels off to an almost constant $\sim 10^{7} \mathrm{erg} \mathrm{g}^{-1}$ value at small target sizes. However, the Flynn \& Durda (2004) experiments only considered a very limited target size range (less than a factor 2 ) in the centimeter domain, so that it seems difficult to conclude to a constant $Q_{s}^{*}(s)$ in the whole $s \leq 1 \mathrm{~cm}$ domain.

Unfortunately, to our knowledge, no experimental data are available for high-velocity collisions of micron-sized grains. In the absence of such data, one of the most useful laboratory experiments are those by Nagaoka et al. (2014), which considered impacts on targets spanning almost two orders of magnitude in sizes down to the millimeter domain, and at impact velocities up to $\sim 1 \mathrm{~km} \mathrm{~s}^{-1}$. We considered the results displayed in Fig. 7 of that paper as a reference for deriving a power-law prescription for $Q_{s}^{*}(s)$, which we then extrapolated down to the sub-micron domain and up to the transition size where gravity takes over. For the gravity regime, we still followed the prescription by Benz \& Asphaug (1999). We also included a simplified dependence on impact velocity derived from Stewart \& Leinhardt (2009), so that our full $Q^{*}(\mathrm{~s})$ prescription reads

$Q^{*}=Q_{0}\left(\frac{s}{1 \mathrm{~cm}}\right)^{-0.4}\left(\frac{v_{\text {coll }}}{v_{1}}\right)^{0.5}+B \rho\left(\frac{s}{1 \mathrm{~cm}}\right)^{1.36}\left(\frac{v_{\text {coll }}}{v_{2}}\right)^{0.5}$,

with $Q_{0}=7 \times 10^{6} \mathrm{erg} \mathrm{g}^{-1}, v_{1}=2.5 \times 10^{4} \mathrm{~cm} \mathrm{~s}^{-1}$ is the typical velocity of the Nagaoka et al. (2014) experiments, $v_{2}=3 \times$ $10^{5} \mathrm{~cm} \mathrm{~s}^{-1}$, and $B=0.3 \mathrm{erg} \mathrm{cm}^{3} \mathrm{~g}^{-2}$.

For both fragmenting and cratering regimes, the size of the largest fragment and the size distributions of the other debris were then derived through the energy scaling prescriptions presented in Thebault et al. (2003) and Thebault \& Augereau (2007). Following Thebault (2016), we also implemented a surface energy-conservation criterion derived from Krijt \& Kama (2014) that gives the lower limit for fragment sizes after any collision.

\subsection{Setup}

We considered the most generic possible case of a classical debris disc at collisional equilibrium and explored three crucial parameters for the problem at hand: stellar type, disc location, and total disc mass. In order to keep the parameter exploration to a manageable level, we considered two representative configurations for each of these parameters. For the stellar type, we considered a $\beta$-Pic like A6V type and a solar-type G2V star. For the disc location, we considered the case of a cold and a warm belt. The cold belt extends from $r_{\text {in }}=50$ to $r_{\text {out }}=90 \mathrm{AU}$ for the A6V case and from 30 to 50 AU (roughly matching a Kuiper belt) for the G2V star. As for the warm belt, it extends from 5 to 9 au for the A6V case and from 3 to $5 \mathrm{AU}$ (roughly matching an asteroid belt) for the G2V star. These radial extents are those of the main parent body disc where all the mass is initially located, but we also simulated the evolution of the much more extended halo of small grains that are produced in this main disc, but are pushed to larger radial distances by radiation pressure (either on highly eccentric bound orbits or by direct blow-out on unbound trajectories).

For the total disc mass, we chose to parametrise it by the IR fractional luminosity of the disc, $f_{\mathrm{d}}$. Because the presence of unbound grains is expected to be favoured in bright and massive

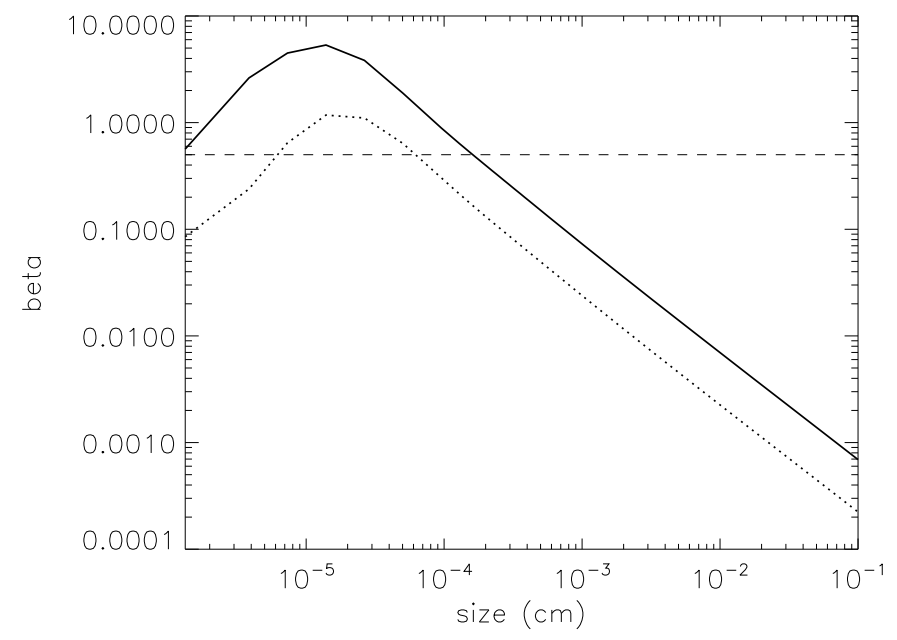

Fig. 1. Value of $\beta$, the ratio between radiation pressure and gravitational forces, as a function of particle size, computed for non-porous astrosilicates. The $\beta(\mathrm{s})$ curve is shown for a luminous A6V star (solid) and for a solar type G2V star (dotted). The dashed horizontal line marks the $\beta=0.5$ limit, above which grains are no longer on bound orbits around the star (if produced from progenitors on circular orbits).

Table 1. Set-up summary.

\begin{tabular}{lc}
\hline \hline Stellar-type & A6V or G2V \\
\hline Material & Compact astrosilicate \\
Blow-out size ( $\left.s_{\text {blow }}\right)$ & $1.6 \mu \mathrm{m}$ (A6V star) \\
& $0.4 \mu \mathrm{m}(\mathrm{G} 2 \mathrm{~V}$ star) \\
Minimum particle size & $0.02 \mu \mathrm{m}$ \\
Maximum particle size & $2 \mathrm{~km}$ \\
Initial size distribution & $\mathrm{d} N \propto s^{-3.5} \mathrm{~d} s$ \\
Dynamical excitation & $\langle e\rangle=2\langle i\rangle=0.075$ \\
Radial extent/cold disc case & $50-90 \mathrm{au}(\mathrm{A} 6 \mathrm{~V}$ star) \\
& $30-50 \mathrm{au}(\mathrm{G} 2 \mathrm{~V}$ star) \\
Radial extent/warm disc case & $5-9 \mathrm{au}$ (A6V star) \\
& $3-5 \mathrm{au}(\mathrm{G} 2 \mathrm{~V}$ star) \\
Fractional luminosity at steady state & $f_{\mathrm{d}} \sim 10^{-3}$ ("bright" disc) \\
& $f_{\mathrm{d}} \sim 5 \times 10^{-3}$ ("very bright" disc) \\
\hline
\end{tabular}

discs (see Sect. 1), we considered the case of a typical "bright" disc with $f_{\mathrm{d}} \sim 10^{-3}$, similar to the archetypal $\beta$-Pictoris system (Decin et al. 2000), and a "very bright" disc with $f_{\mathrm{d}} \sim 5 \times 10^{-3}$, such as the HR 4796 (Jura et al. 1998) or HD 32297 (Moor et al. 2006) systems. These $f_{\mathrm{d}}$ values are not necessarily the values at $t=0$ but are the values when a steady state is reached. In practice, we always started with discs whose $f_{\mathrm{d}}$ was larger than we were aiming for, and let the systems collisionally evolve until (1) the shape of the particle size distribution (PSD) no longer changed (steady state), and (2) $f_{\mathrm{d}}$ decreased to the desired value. For the dynamical excitation of the disc, we chose a standard configuration with $\langle e\rangle=2\langle i\rangle=0.075$ (Thebault 2009).

The problem we address is very specific, therefore we considered the evolution of the PSD from $s_{\max }=2 \mathrm{~km}$ down to a very small size of $s_{\min }=0.02 \mu \mathrm{m}$. For dust material, we considered a standard case of compact astrosilicates (Draine 2003). Figure 1 displays the $\beta$ (s) curves for this material and the two stellar types considered here.

All main parameters are summarised in Table 1. 


\section{Results}

\subsection{A-type star}

\subsubsection{Cold outer belt}

Figure 2 displays the PSD at collisional steady state for a bright and a very bright disc. The PSDs show well-known features, such as a power-law distribution in $\mathrm{d} N \propto s^{-q} \mathrm{~d} s$ that holds in most of the bound-grains size domain, where $q$ slightly exceeds the canonical 3.5 value because the $Q_{s}^{*}(s)$ prescription depends on size (Gáspár et al. 2012; Kral et al. 2013, and references therein). We also see the well-known waviness in the PSD at the lower end of the bound-grain size domain, which is due to the discontinuity at the $s=s_{\text {blow }}$ frontier (Campo Bagatin et al. 1994; Thebault et al. 2003; Thebault \& Augereau 2007).

When we now focus on the unbound grains domain, we observe, as expected, a drop at the $s=s_{\text {blow }}$ transition. For the $f_{\mathrm{d}}=10^{-3}$ disc, this density drop is very pronounced, close to two orders of magnitude, so that even if the PSD increases again for grains in the $\simeq 0.1 \mu \mathrm{m}$ range, the total geometrical cross section of the disc is largely dominated by bound grains. In contrast, for the very bright $f_{\mathrm{d}}=5 \times 10^{-3}$ disc, the density drop around $s_{\text {blow }}$ is much less pronounced, only of the order of a factor 5. As a consequence, the upturn in the PSD in the $s \leq 0.1 \mu \mathrm{m}$ domain is able to make the smallest grains the dominant contributors to the total cross section (Fig. 2). However, this might not necessarily translate into a significant contribution to the observed flux because these grains might have a low emissivity because they are very small.

To check this, we used the GraTeR package (Augereau et al. 1999) to derive synthetic SEDs (Fig. 3). We also estimated at all wavelengths the relative contribution of unbound grains to the total disc flux (Fig. 4). In order to simulate the case of an unresolved disc, we took the contribution of grains in the extended halo into account. This halo is located beyond the main disc, and its grains have been collisionally produced in the main belt but populate the $r \geq r_{\text {out }}$ region because radiation pressure places them on unbound or high-eccentricity orbits (Strubbe \& Chiang 2006; Thebault \& $\mathrm{Wu} 2008)$. Even for the $f_{\mathrm{d}}=10^{-3}$ disc case where they do not dominate the cross section, unbound grains can significantly contribute to the spectral energy distribution (SED), exceeding $50 \%$ of the flux, but in a relatively narrow domain between $\lambda \sim 10$ and $\sim 20 \mu \mathrm{m}$ (Fig. 4a). This is partly due to the well-known silicate band around $\sim 10 \mu \mathrm{m}$ (see Sect. 4.2). However, the dominant effect is that the $10 \leq \lambda \leq 20 \mu \mathrm{m}$ domain is a sweet spot where the higher temperature of these submicron grains, which exceeds the almost black-body temperature of larger particles, has a dramatic effect. The reason is that at $\sim 70 \mathrm{AU}$ from an A6V star, the $\lambda \leq 20 \mu \mathrm{m}$ domain is deep in the Wien side of the Planck function, for which the flux increases exponentially with temperature. This exponential dependence is strong enough to compensate for the lower emissivity of grains that are smaller than the $s=\lambda / 2 \pi$ limit. Beyond $\lambda \sim 20 \mu \mathrm{m}$, we leave this purely exponential temperature-dependence regime, and the higher temperature of the tiniest grains can no longer mitigate their decreasing emissivity. Below $\lambda \sim 10 \mu \mathrm{m}$, the flux becomes dominated by scattered light, for which the contribution of unbound grains sharply drops before it again increases to $\sim 20 \%$ of the flux at $\lambda \sim 0.5 \mu \mathrm{m}$ (Fig. $4 \mathrm{a}$ ).

For a very bright disc with $f_{\mathrm{d}} \sim 5 \times 10^{-3}$, the contribution of unbound grains is, as expected, even higher. They contribute to more than $50 \%$ of the flux in the $8 \leq \lambda \leq 30 \mu$ m domain, and even up to $80-90 \%$ for $10 \leq \lambda \leq 20 \mu \mathrm{m}$ (Fig. 4b). Moreover, they

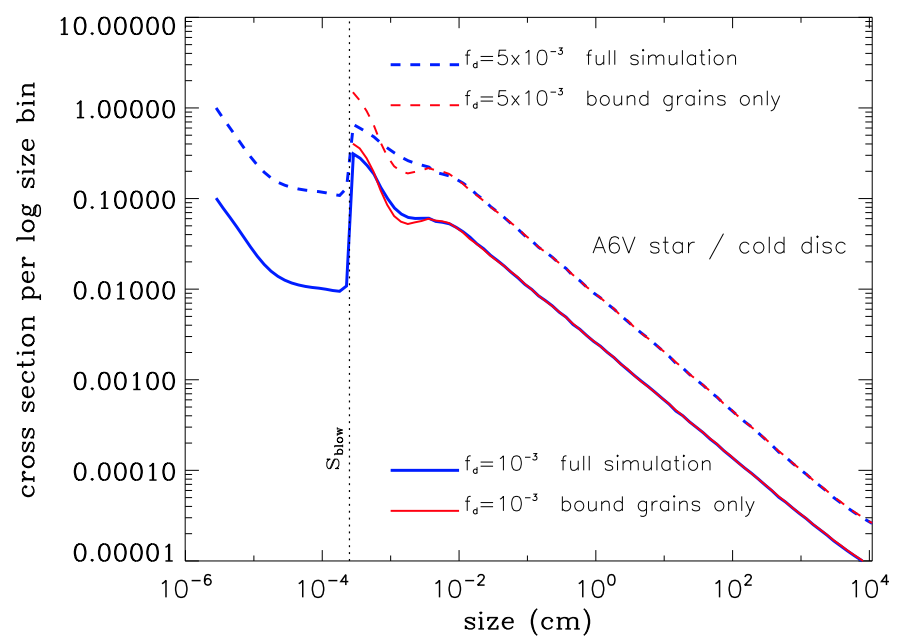

Fig. 2. Cold disc $(50 \leq r \leq 90 \mathrm{au})$ of an A6V star: normalised discintegrated particle size distribution at collisional steady state for a "bright" debris disc with $f_{\mathrm{d}} \sim 10^{-3}$, and a "very bright" debris disc with $f_{\mathrm{d}} \sim 5 \times 10^{-3}$. The red line corresponds to a simulation that only takes $s \geq s_{\text {blow }}$ grains into account. The vertical dotted line corresponds to the blow-out size $s_{\text {blow }}$.

also contribute to more than $30 \%$ of the scattered-light flux at all wavelengths shorter than $\sim 3 \mu \mathrm{m}$.

Unbound grains can also affect the disc evolution indirectly, by collisonally eroding the population of small bound particles above the $s_{\text {blow }}$ limit. This effect was first noted in the avalanche simulations of Thebault \& Kral (2018). To assess the importance of this effect, we performed additional simulations where only bound $s \geq s_{\text {blow }}$ grains were taken into account and compared the obtained PSDs and SEDs to those obtained for our full simulations ${ }^{4}$. For the bright $f_{\mathrm{d}}=10^{-3}$ disc, the effect is already noticeable on the PSD, but remains marginal. It is for the very bright disc case that the eroding effect leaves a very pronounced signature on the PSD, with a deficit of at least a factor 2 of small bound grains in the full simulation compared to the bound-grains-only run (Fig. 2).

Another important result is that regardless of the disc mass or brightness, the halo beyond the main belt contributes significantly to the flux by $\sim 50 \%$ at almost all wavelengths $\lambda \lesssim 50 \mu \mathrm{m}$ (Fig. 4). This contribution then drops beyond $\lambda \sim 50 \mu \mathrm{m}$ because the halo only contains grains smaller than $s \sim 10-20 \mu \mathrm{m}$, which are poor emitters at these long wavelengths.

\subsubsection{Warm inner belt}

The effect of unbound grains on the PSD is stronger when an asteroid-like warm disc ( $5 \leq r \leq 9 \mathrm{au})$ is considered. Unbound

\footnotetext{
4 For these bound-only simulations, we took the same number density for large parent bodies $(s>1 \mathrm{~m}$ ), that is, the same disc mass, as for the corresponding full simulation. As a result, the steady state $f_{\mathrm{d}}$ of these bound-only systems can in principle differ from that of the full simulation because of the absence of unbound grains and different number densities of particles close to the $s_{\text {blow }}$ limit. However, despite significant flux differences in some specific narrow wavelength domains (e.g. around $\lambda \sim 10-20 \mu \mathrm{m}$ ), the differences in global $f_{\mathrm{d}}$ always remain relatively limited (lower than $\sim 25 \%$ ), mainly because the drop in $f_{\mathrm{d}}$ due to the absence of unbound dust is to a large extent compensated for by the $f_{\mathrm{d}}$ increase that is due to the excess of small bound grains that are not destroyed by these absent sub-micron grains. For the sake of simplicity and readability, we thus chose to label both the full simulation and its bound-only counterpart with the $f_{\mathrm{d}}$ value of the full simulation.
} 

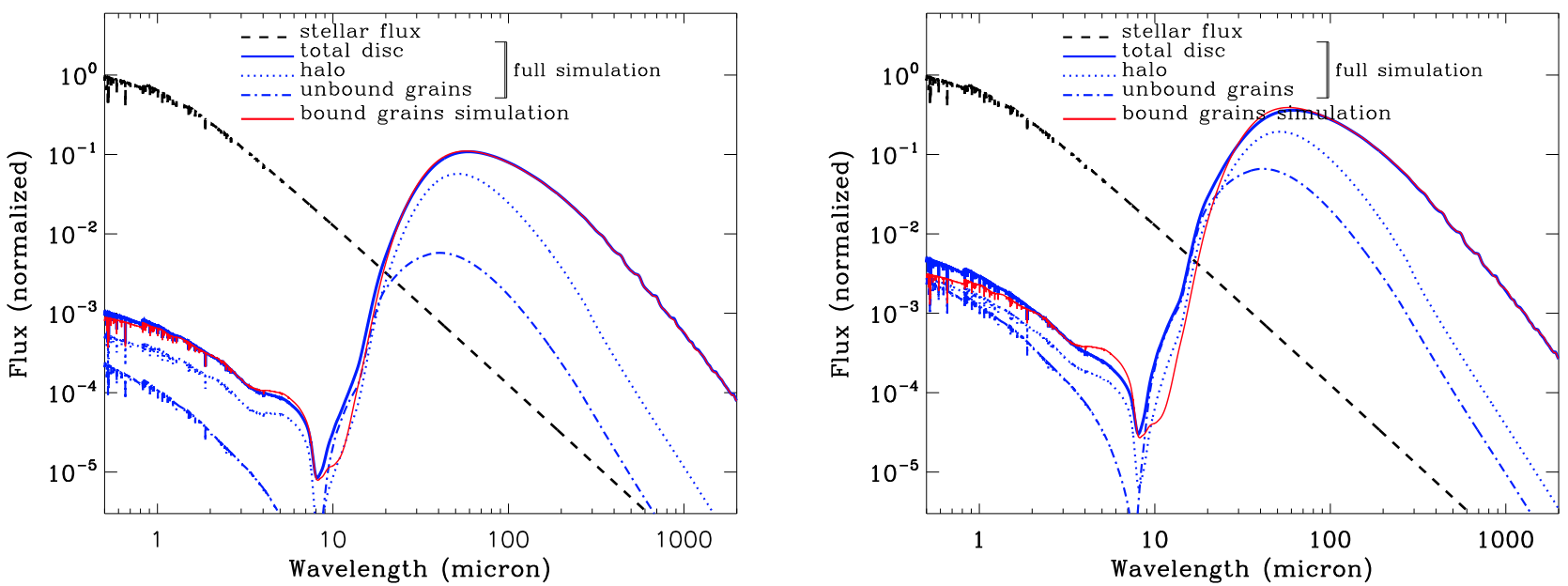

Fig. 3. Cold disk of an A6V star: normalised SED (solid line in blue). Left: bright debris disc with $f_{\mathrm{d}} \sim 10^{-3}$. Right: very bright debris disc with $f_{\mathrm{d}} \sim 5 \times 10^{-3}$. The dash-dotted blue line shows the contribution of unbound $s \leq s_{\text {blow }}$ grains. The dotted blue line represents the contribution of grains in the halo beyond the main disc. The red line corresponds to a simulation that only takes $s \geq s_{\text {blow }}$ grains into account.
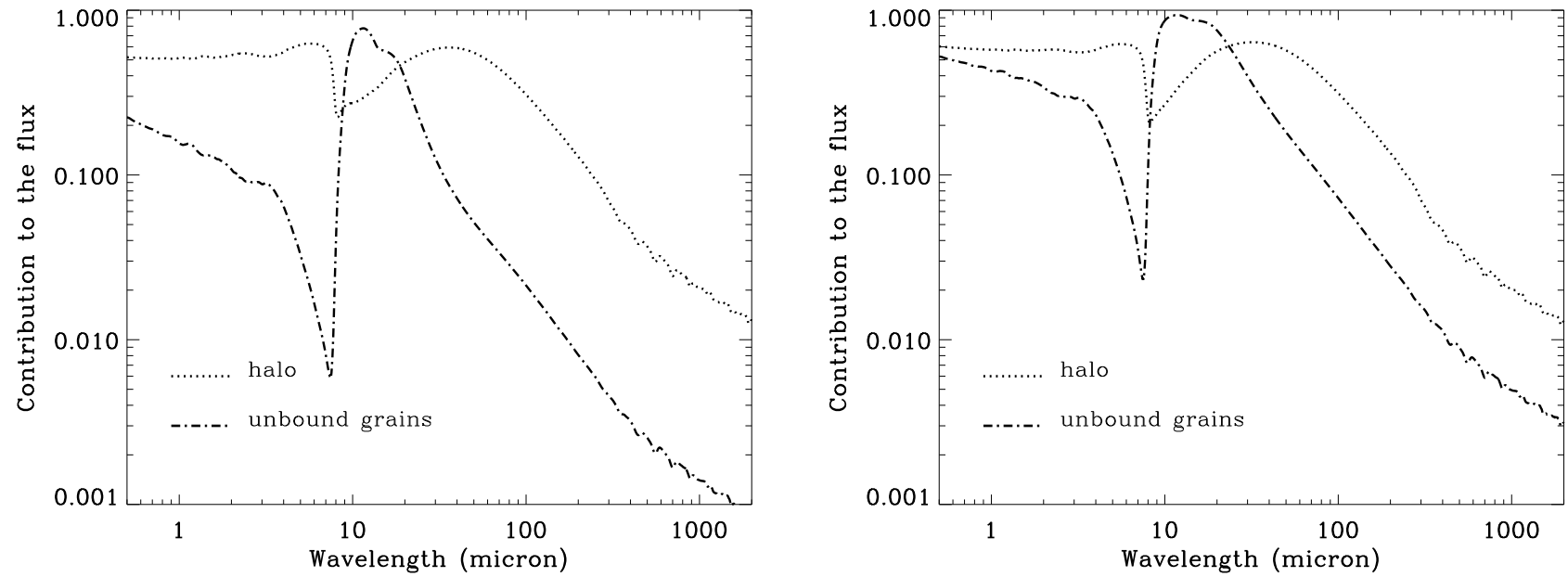

Fig. 4. Cold disc of an A6V star: fractional contribution of unbound (dash-dotted line) and halo (dotted line) grains to the SED as a function of wavelength. Left: bright debris disc with $f_{\mathrm{d}} \sim 10^{-3}$. Right: very bright debris disc with $f_{\mathrm{d}} \sim 5 \times 10^{-3}$.

grains leave a strong signature even for a disc with a fractional luminosity $f_{\mathrm{d}} \sim 10^{-3}$, for which we see (Fig. 5) that the collisional cross section is clearly dominated by $s \leq s_{\text {blow }}$ grains. The collisional erosion of small bound particles due to these unbound grains is more efficient than in the cold disc case, and it can reduce the number density of $s_{\text {blow }} \leq s \leq 2 s_{\text {blow }}$ grains by a factor of $\sim 5$. As expected, the effect of unbound grains becomes even stronger for a brighter $f_{\mathrm{d}}=5 \times 10^{-3}$ disc, for which the density drop at the $s_{\text {blow }}$ frontier is almost completely erased. The explanation for this stronger effect of unbound grains for the same $f_{\mathrm{d}}$ is that impact velocities, and thus the collisional activity, are higher here than in a cold disc, which is located beyond $50 \mathrm{au}^{5}$.

As expected, the unbound grains also leave a significant imprint on the SEDs (Fig. 6), but in a different way as for the cold disc case. The peak due to the silicate band at $\sim 10 \mu \mathrm{m}$

5 The absolute collision rates are also higher for the same $f_{\mathrm{d}}$ than for a cold disc. However, what matters for the amplitude of the drop at $s_{\text {blow }}$ is the ratio between the collisional and the dynamical (i.e. orbital) timescales, and this ratio is inversely proportional to the normal geometrical depth $\tau$ and thus to $f_{\mathrm{d}}$ (Zuckerman \& Song 2012).

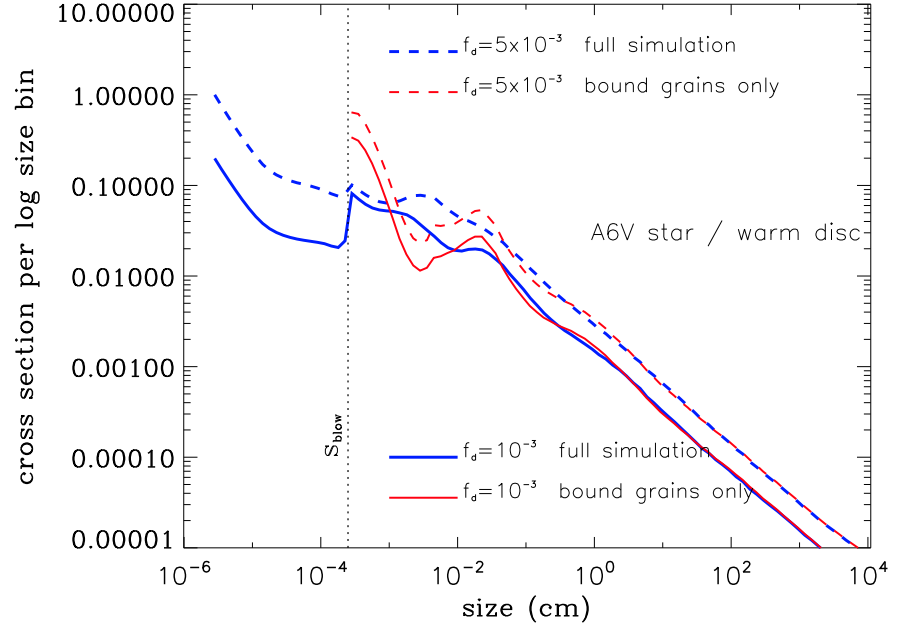

Fig. 5. Same as Fig. 2, but for a warm disc $(5 \leq r \leq 9 \mathrm{au})$.

is much more prominent here and clearly dominates the contribution of the thermal continuum in the wavelength domain. This is both because the peak amplitude is directly proportional 

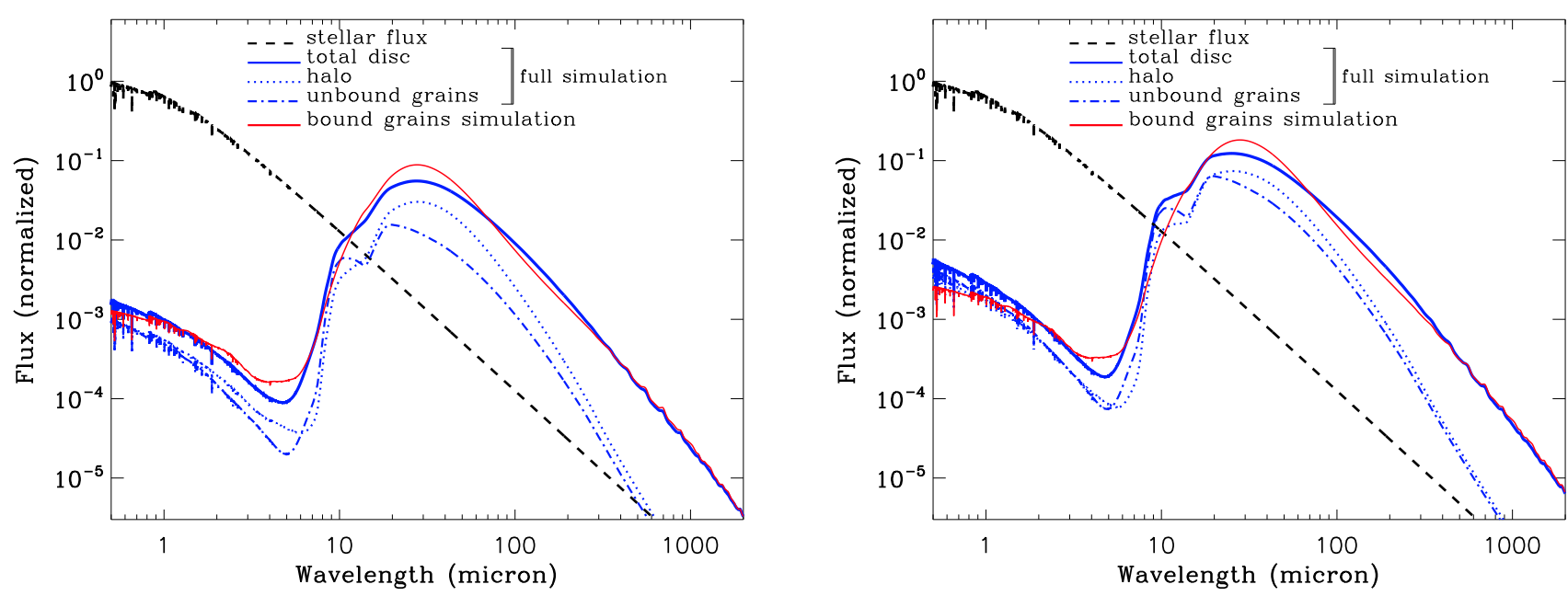

Fig. 6. Same as Fig. 3, but for a warm disc $\left(5 \leq r \leq 9\right.$ au) with $f_{\mathrm{d}}=10^{-3}$ (left panel) and $f_{\mathrm{d}}=5 \times 10^{-3}$ (right panel).
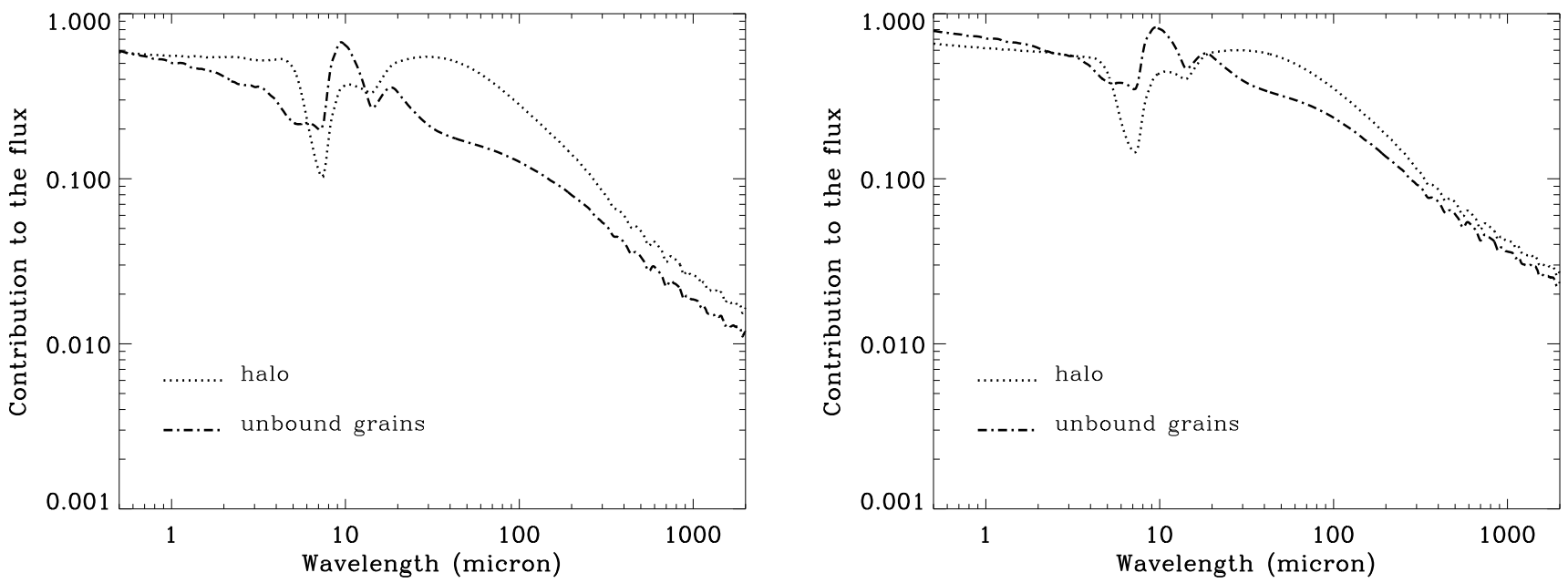

Fig. 7. Same as Fig. 4, but for a warm disc $\left(5 \leq r \leq 9\right.$ au) with $f_{\mathrm{d}}=10^{-3}$ (left panel) and $f_{\mathrm{d}}=5 \times 10^{-3}$ (right panel).

to the number density of unbound grains, which is higher than in the cold disc case, and because the Wien continuum in the $\lambda \sim 10-15 \mu \mathrm{m}$ domain is less steep for this warmer case, making the feature easier to discern. The depletion of small bound grains in the $s \sim 1-5 \mu \mathrm{m}$ size domain results in a flux deficit in the $\lambda \sim 15-40 \mu \mathrm{m}$ range, which combined with the aforementioned bump around $\lambda \sim 8-15 \mu \mathrm{m}$ leaves a much flatter SED in the whole $\lambda \sim 8-40 \mu \mathrm{m}$ domain than in the bound-grains-only simulation (red curve on Fig. 6).

\subsection{Solar-type star}

A solar-type star changes two important things. Firstly, the blowout size is smaller, around $s_{\text {blow }} \sim 0.4 \mu \mathrm{m}$, and secondly, there is a second critical "blow-out" size $s_{\text {blow } 2} \sim 0.07 \mu \mathrm{m}$ below which the particle orbits become bound again (Fig. 1).

\subsubsection{Cold outer belt}

Figure 8 shows that the PSD is schematically divided into three different domains: a classical power-law profile (with a slight waviness at its lower end) down to $s_{\text {blow }}$, followed by a sharp drop and an almost plateau value in the $s_{\text {blow } 2} \leq s \leq s_{\text {blow }}$ interval, and finally, a very strong upturn for $s \leq s_{\text {blow2 }}$ grains because

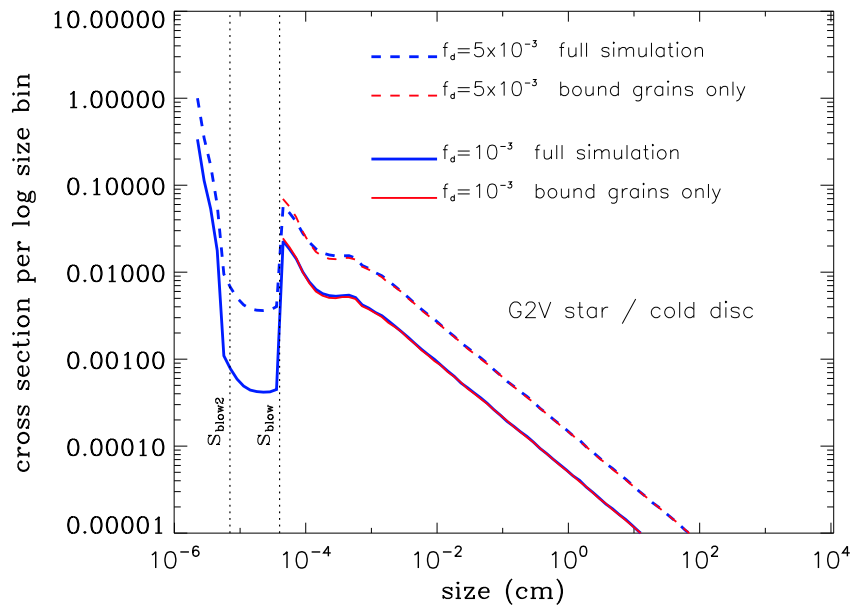

Fig. 8. Same as Fig. 2, but for a solar-type G2V star. The vertical dotted lines correspond to the two blow-out size limits $s_{\text {blow }}$ and $s_{\text {blow2 }}$.

these grains are no longer blown out of the system and remain on bound orbits. This upturn is strong enough for the total cross section of the system to be dominated at more than $90 \%$ by these $s \leq s_{\text {blow2 }}$ particles, and this even in the $f_{\mathrm{d}}=10^{-3}$ disc case. 

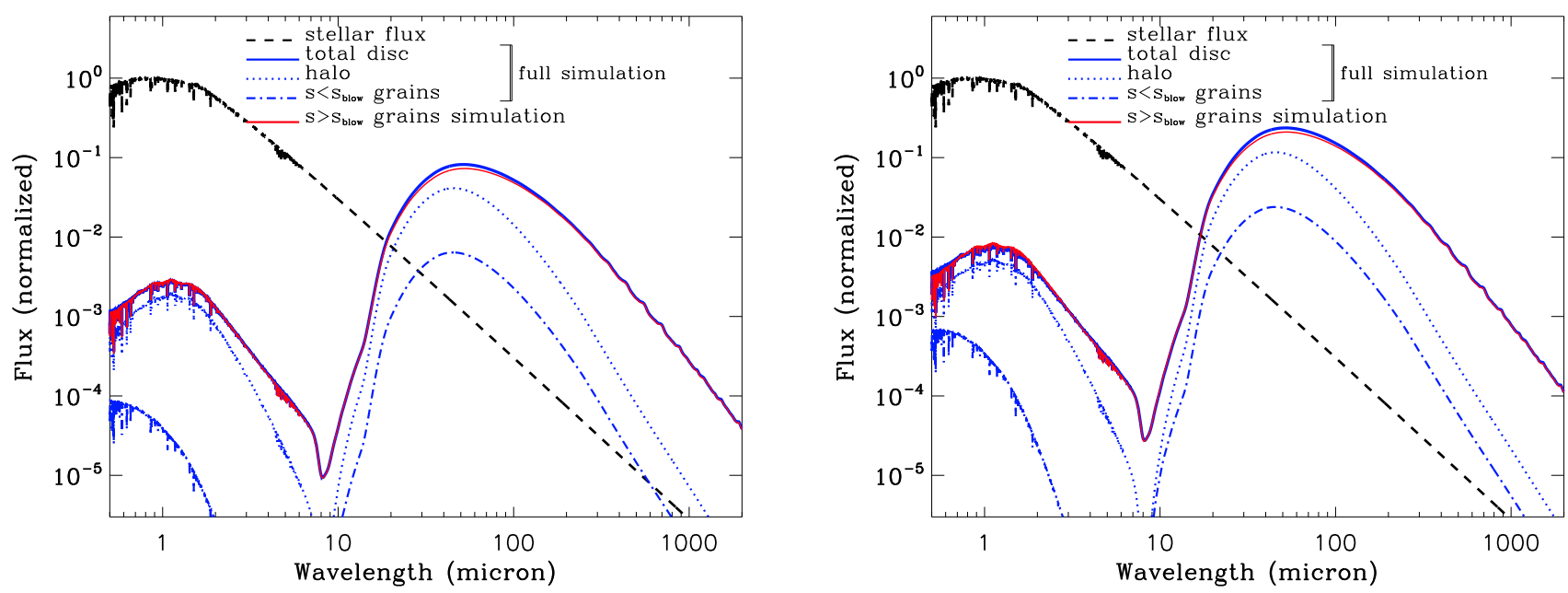

Fig. 9. Same as Fig. 3, but for a solar-type G2V star with $f_{\mathrm{d}}=10^{-3}$ (left panel) and $f_{\mathrm{d}}=5 \times 10^{-3}$ (right panel).

However, this vast population of tiny grains has a very limited erosion effect on the population of small bound grains, with the $s \geq s_{\text {blow }}$ PSD staying very close to what it is in a bound-grainsonly simulation. The reason is that the bound orbits of these tiny grains cause them to impact larger grains at much lower velocities than in the case of an A star that blows them away on high-speed outbound trajectories.

Furthermore, the $s \leq s_{\text {blow2 }}$ grain dominance over the cross section does not translate into a significant effect on the SED (Fig. 9), with a contribution that almost never exceeds $10 \%$ of the flux at any wavelengths, even for a dense very bright $f_{\mathrm{d}}=5 \times 10^{-3}$ disc. The higher temperature of these grains is here not able to compensate for their extremely small emission coefficient because of their very small size. While the silicate band is clearly noticeable around $11 \mu \mathrm{m}$, it is mostly due to $s \geq s_{\text {blow }}$ grains because the lower value of the $s_{\text {blow }}$ limit allows for submicron grains to contribute to it even in a bound-grains-only simulation.

\subsubsection{Warm inner belt}

The situation does not radically change for a warm asteroidlike belt. The only difference is that because of higher impact velocities, the erosion of small bound grains by the tiniest particles is now noticeable on the PSD (Fig. 10). However, the effect on the SED remains relatively limited and is only detected as a scattered-light flux deficit (the missing contribution of the small bound grains is here, in contrast to the A-star case, not compensated for by the contributions of the unbound grains), and a flatter SED in the $\sim 0.7-2 \mu \mathrm{m}$ domain.

\section{Discussion}

Our simulations have shown that bright debris discs (with $f_{\mathrm{d}} \gtrsim$ $10^{-3}$ ) at collisional steady state are able to naturally produce a significant amount of small $s \leq s_{\text {blow }}$ unbound grains, without the need to invoke transient and/or violent processes such as the breakup of large planetesimals or collisional avalanches. In addition, these grains can also significantly erode the population of small bound grains in the $s_{\text {blow }} \leq s \lesssim 2-3 s_{\text {blow }}$ domain.

For solar-type stars, $s \leq s_{\text {blow }}$ grains can account for at least $90 \%$ of the geometrical cross section of the disc, but leave a marginal signature on the SED. For bright A-type stars, however, these unbound grains, even if they contribute less to the total

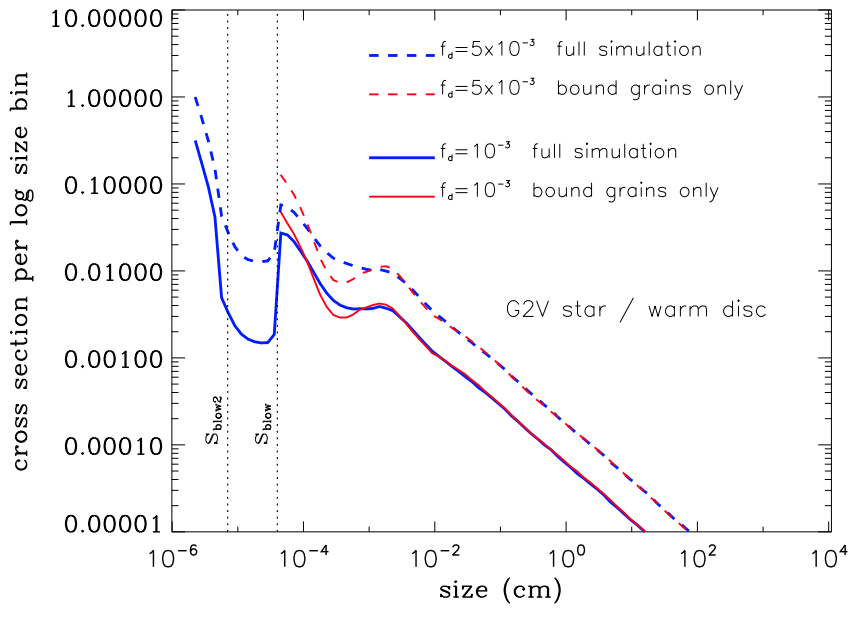

Fig. 10. Same as Fig. 2, but for a solar-type G2V star. The vertical dotted lines correspond to the two blow-out size limits $s_{\text {blow }}$ and $s_{\text {blow2 }}$.

cross section, can leave a much more significant imprint on the SED. This is especially true in two wavelength domains: in scattered light in the visible to near-IR $(0.5 \lesssim \lambda \lesssim 2 \mu \mathrm{m})$, and in thermal emission in the mid-IR $(10 \lesssim \lambda \lesssim 20-30 \mu \mathrm{m})$.

We now discuss to what extent these results can be compared to observational constraints. Exploring the specificities of several individual debris discs would exceed the scope of this paper, and we instead investigate whether our simulations can explain some general characteristics or trends that have been observationally derived for bright debris discs. We distinguish two main categories of observationally derived features: those that have been attributed to sub-micron grains without a satisfying physical explanation for their presence or with explanations involving transient events (blue colour, silicate feature), and features that have so far been explained without resorting to blow-out grains but might at least in some cases be attributed to them when our results here are considered (high mid-IR brightness, double belts).

\subsection{Mid-IR flux}

Our results show that for an A-star, $s \leq s_{\text {blow }}$ grains dominate the flux in the $10-20 \mu \mathrm{m}$ wavelength domain for bright discs with $f_{\mathrm{d}} \geq 10^{-3}$. This is a relatively counter-intuitive result 

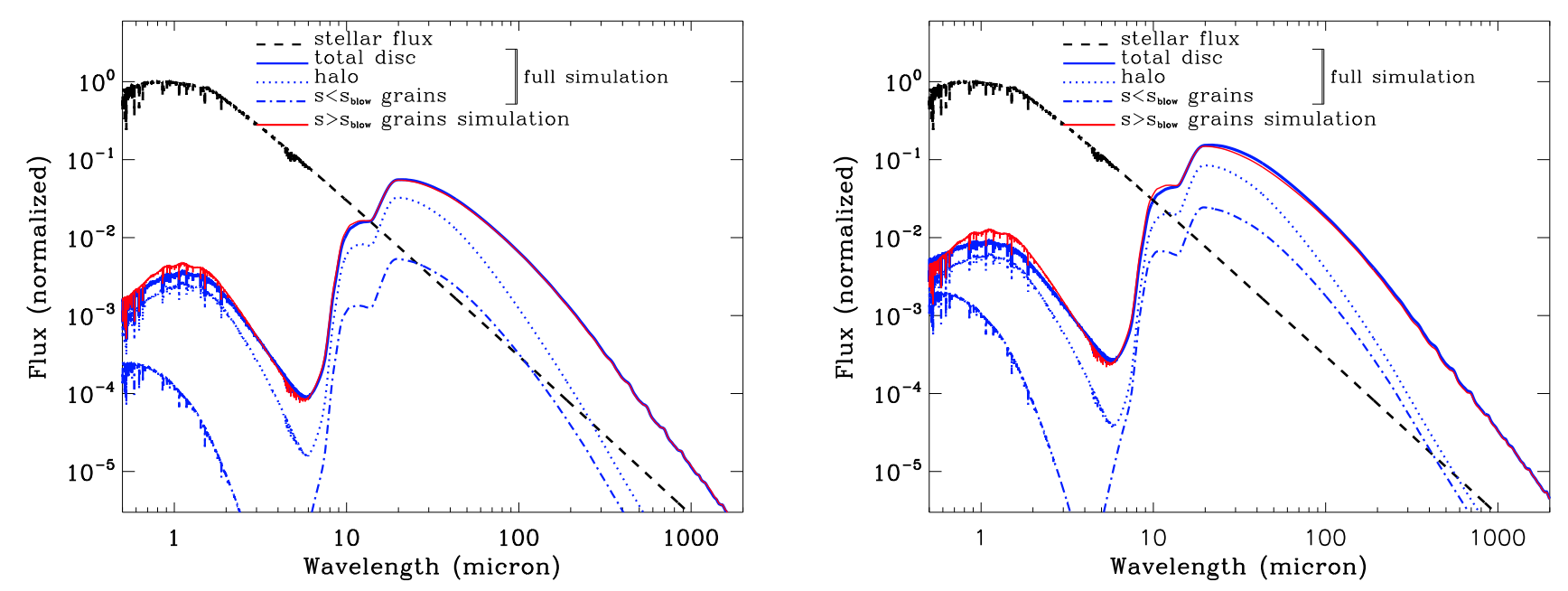

Fig. 11. Same as Fig. 6, but for a solar-type G2V star with $f_{\mathrm{d}}=10^{-3}$ (left panel) and $f_{\mathrm{d}}=5 \times 10^{-3}$ (right panel).

because these wavelengths are much longer than $2 \pi s$. For warm discs $(5 \leq r \leq 9 \mathrm{AU})$, this is primarily because these sub-micron grains dominate the total geometrical section $\Sigma$ (Fig. 5) and because this dominance is strong enough to compensate for their lower emissivity at these wavelengths. For cold discs, submicron grains do not dominate $\Sigma$, but their higher temperature ( $\sim 115 \mathrm{~K}$ instead of $\sim 80 \mathrm{~K}$ for larger grains) results in an exponential increase of the Planck function (because we are deep in the Wien part of the function), which compensates for both their lower $\Sigma$ and their lower absorption efficiency $Q_{\mathrm{abs}}$. This added luminosity due to unbound grains also causes the discs to appear brighter in the mid-IR by at least a factor 2 than what would be expected otherwise. This is especially interesting for cold discs, which might become detectable in the mid-IR although they are located far beyond the stellar distances for which the thermal emission is expected to peak around $10-20 \mu \mathrm{m}$. This could, for example, provide a natural explanation for the observation of the bright mid-IR clump at 50-70 AU from $\beta$ Pictoris by Telesco et al. (2005), especially because this clump seems to contain a significant population of sub-micron grains.

\subsection{Silicate band at $10 \mu \mathrm{m}$}

Another consequence of the strong contributions of unbound grains to the mid-IR flux is that the silicate line around $10 \mu \mathrm{m}$ becomes detectable in the SED. This is especially true for the warm disc case, where it clearly appears in addition to the continuum (Fig. 12b). This is not a surprising result in itself because this silicate line has been repeatedly observed in several warm or hot discs and was often interpreted as a sign for the presence of sub-micron silicate grains (Matthews et al. 2014), being due to a surge of the absorption coefficient $Q_{\text {abs }}$ at these wavelengths (attributed to stretching of the $\mathrm{Si}-\mathrm{O}$ bonds) for grains of sizes $s \lesssim 2 \mu \mathrm{m}$ (Voshchinnikov \& Henning 2008). This has for instance been the case for HD 69830 (Beichman et al. 2005; Lisse et al. 2007), HD 32297, HD 113766 (Lisse et al. 2008), HD 172555 (Lisse et al. 2009; Johnson et al. 2012), or BD+020 307 (Weinberger et al. 2011). However, these observational studies were often not able to place constrains on the quantity of sub-micron grains associated with the lines, or if they did, it was without exploring possible physical explanations for their presence. We are here for the first time able to show that for bright warm discs around early-type stars, the steady-state collisional evolution of the system naturally produces enough sub-micron astrosilicate grains for the $10 \mu \mathrm{m}$ line to appear. Some additional exploratory runs showed that the $10 \mu \mathrm{m}$ line remains detectable down to disc fractional luminosities $f_{\mathrm{d}} \sim$ $5 \times 10^{-4}$.

The $10 \mu \mathrm{m}$ feature being due to a variation in $Q_{\mathrm{abs}}$, it is in principle independent of temperature. It should therefore also be detectable in cold discs with significant amounts of sub-micron grains. However, even when we subtract the continuum, we fail to clearly identify the feature (Fig. 12a), mainly because scattered light contributes dominantly to the flux up to the $\sim 10 \mu \mathrm{m}$ domain and drowns a large part of the signal due to the silicate emission line. The right-hand side of the silicate line is visible in Fig. 12a, but quickly merges into the scattered-light flux at lower wavelengths. Another factor that makes the $10 \mu \mathrm{m}$ line very difficult to detect in cold disc observations is that it corresponds to a wavelength domain where the thermal emission of these distant discs is very low. In addition, even if the thermal flux is detected around $\lambda \sim 10 \mu \mathrm{m}$, its very sharp variation with $\lambda$ makes the silicate feature very difficult to detect in practice. Last but not least, the contribution from even a small amount of hotter dust located closer to the star would completely drown, at $10 \mu \mathrm{m}$, the signal from these outer regions. To our knowledge, the only system where the $10 \mu \mathrm{m}$ silicate band has been identified in a cold belt $(T \lesssim 200 \mathrm{~K})$ is HD 15745 , which Mittal et al. (2015) fitted with grains at $T=156 \mathrm{~K}$.

For warm discs around solar-type stars, the silicate line is clearly visible in our synthetic spectra, even before we subtract the continuum (Fig. 11). However, small unbound grains only marginally contribute to it, mainly because the blow-out size $s_{\text {blow }}$ is already well into the $s \lesssim 2 \mu \mathrm{m}$ domain, so that the abundant bound grains close to $\sim s_{\text {blow }}$ can produce a strong silicate feature. This result is in agreement with the fact that the $10 \mu \mathrm{m}$ silicate feature is more readily observed around $\mathrm{G}$ stars than earlier-type stars (Matthews et al. 2014).

We note, however, that the observation of a clear silicate feature at $\lambda \sim 10 \mu \mathrm{m}$ is not always interpreted as a clear sign for the presence of sub-micron grains. The strength, peak position, and shape of the silicate band is indeed sensitive to the exact physical compositions of the grains, such as the mix between different types of silicates, mainly olivine $\left(\mathrm{Mg}_{2 x} \mathrm{Fe}_{2-2 x} \mathrm{SiO}_{4}\right)$ or pyroxene $\left(\mathrm{Mg}_{x} \mathrm{Fe}_{1-x} \mathrm{SiO}_{3}\right)$, the fraction $x$ of $\mathrm{Mg}$ and $\mathrm{Fe}$ in their 

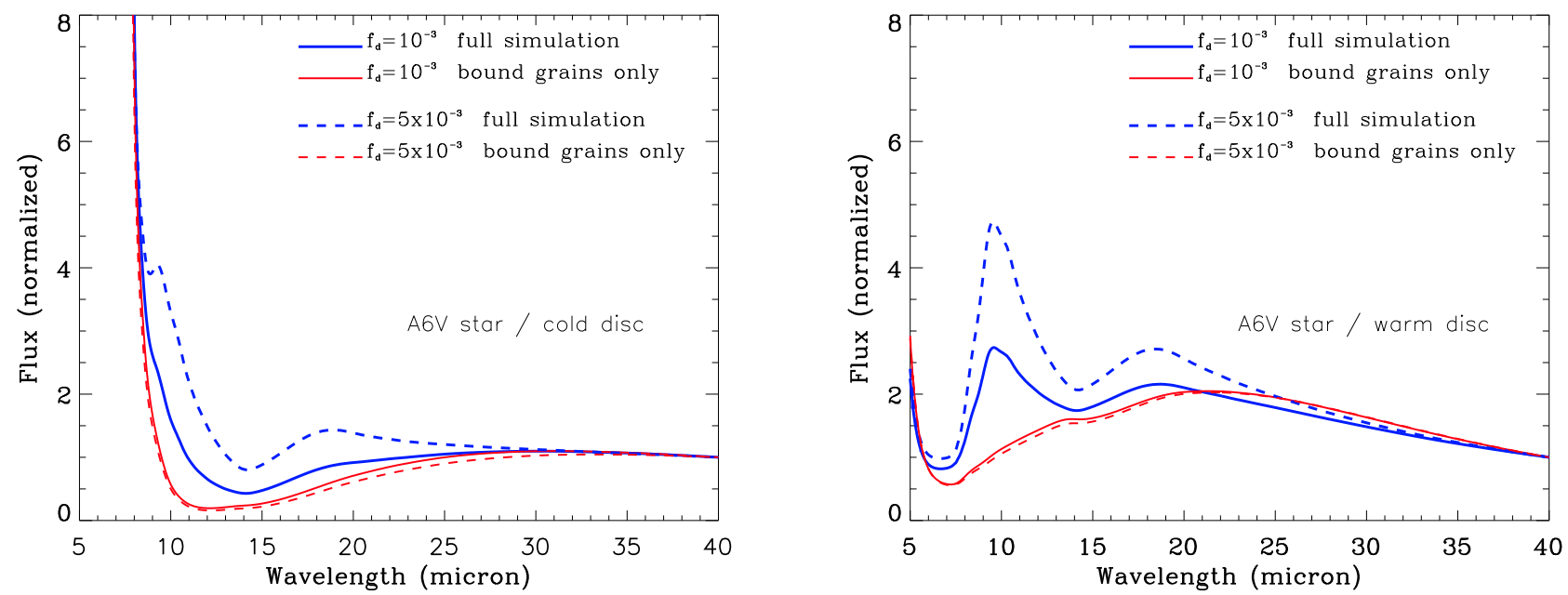

Fig. 12. A6V star runs. Mid-IR flux divided by the black-body continuum extrapolated from the flux at $\lambda=40 \mu \mathrm{m}$ assuming $T=115 \mathrm{~K}$ for the cold disc case, and $T=275 \mathrm{~K}$ for the warm disc case.
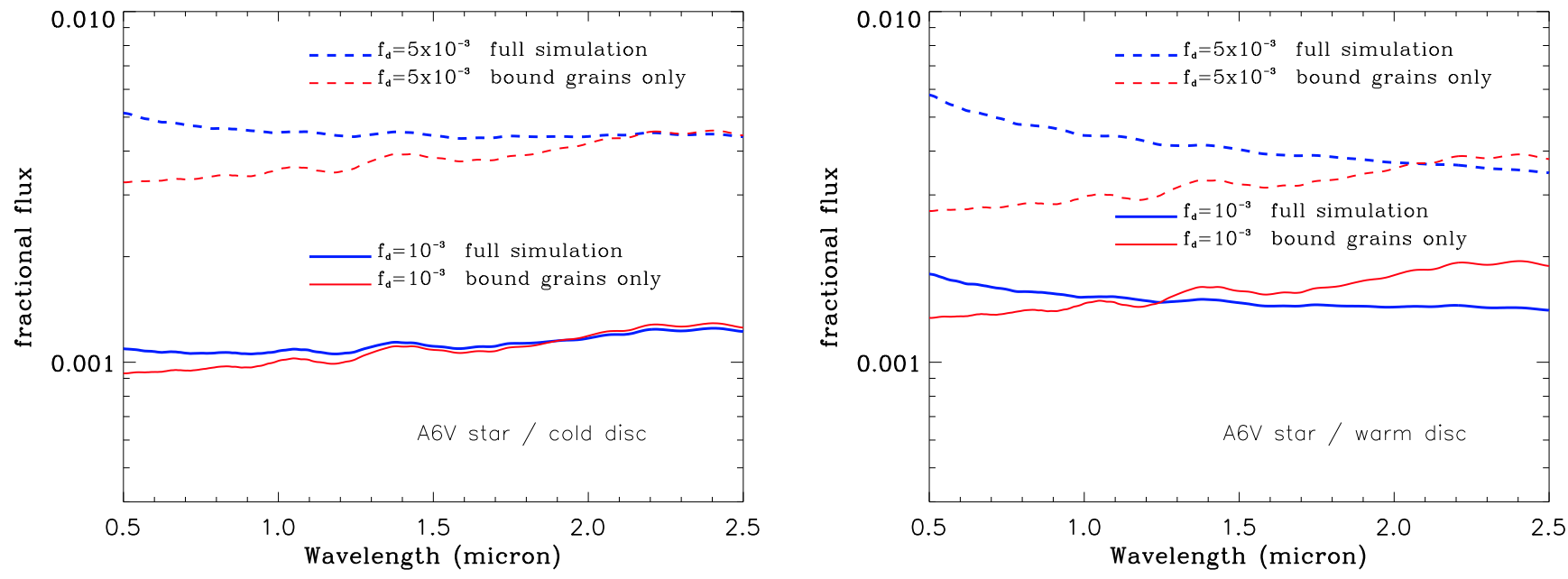

Fig. 13. A6V star runs: ratio of the scattered-light flux of the disc to the stellar flux in the near-IR.

composition, or the balance between amorphous and crystaline components (Draine 2003; Henning 2010; Olofsson et al. 2010). This probably explains why some studies have been able to fit the observed mid-IR spectra (including the $10 \mu \mathrm{m}$ feature) of several discs with silicate grains whose PSD does not extend far below the blow-out size $s_{\text {blow }}$ (e.g. Ballering et al. 2014; Mittal et al. 2015). However, it is important to stress that these fitting procedures relied on very simplified assumptions for the PSD, notably the absence of any discontinuity at the $s=s_{\text {blow }}$ limit, which made it very difficult to take the potential effect of unbound grains into account in a reliable way.

\subsection{Colour index}

Another observationally derived clear sign of the presence of sub-micron grains is a blue colour of the spectrum when it is divided by the stellar contribution in the visible to near-IR range. It is indeed expected that very small sub-micron grains (bound or unbound) Rayleigh scatter and are therefore blue, while grains larger than a few micron scatter neutrally and grains in between would appear red (e.g. Meyer et al. 2007). A handful of systems with a blue scattered-light colour are known to date, such as HD 32297 (Kalas 2005), HD 61005 (Esposito et al. 2016), the blue needle HD 15115 (Debes et al. 2008b), and the famous AU Mic (Lomax et al. 2018). As with the $10 \mu \mathrm{m}$ silicate band, the amount of sub-micron grains required to explain the observed blue colours is often not quantified, or if it is, it is by using radiative transfer models assuming unrealistically continuous particle size distributions that smoothly cross the $s_{\text {blow }}$ boundary. In most cases, the level of this unbound grain contribution is identified as a puzzling feature that has been interpreted as the consequence of violent and/or transient events (with the notable exception of Fitzgerald et al. 2007a, who mentioned the potential role of grain composition and/or porosity).

We showed for the first time that for A-type stars, a bright debris disc at collisional steady state can naturally produce enough sub-micron grains to induce a blue colour in scattered light. This is clearly illustrated in Figs. 13 and 14, which show the slope of the fractional $F_{\text {disc }} / F_{*}$ luminosity in the $0.5-2.5 \mu \mathrm{m}$ range. For all A star cases except for the cold disc case with $f_{\mathrm{d}}=10^{-3}$, the scattered-light spectrum turns from red to blue when the effect of unbound grains is taken into account. Even in the remaining cold disc with $f_{\mathrm{d}}=10^{-3}$, the disc is still significantly bluer than in a run that stops at the $s=s_{\text {blow }}$ boundary. We note that of the few systems with observed blue colours, two (HD 32297 and HD 15115) correspond to bright discs 

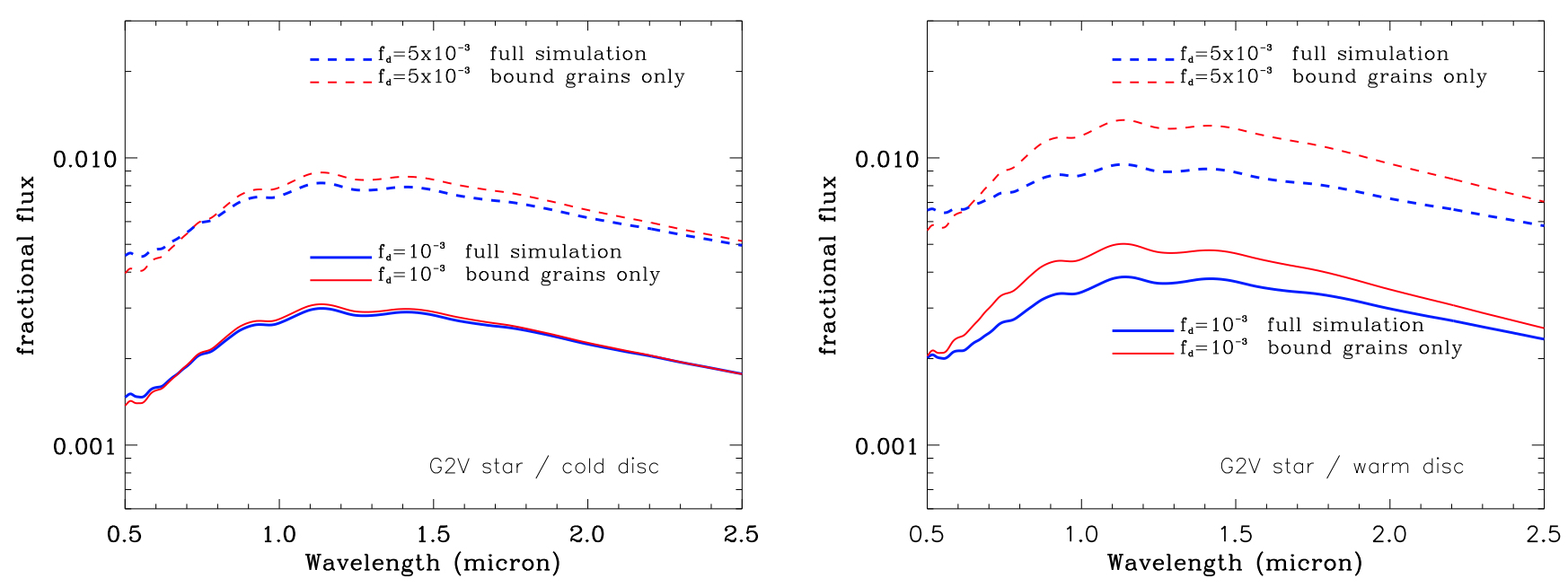

Fig. 14. G2V star runs: ratio of the scattered-light flux of the disc to the stellar flux in the near-IR.

$\left(f_{\mathrm{d}} \geq 10^{-3}\right)$ around $\mathrm{A}$ or $\mathrm{F}$ stars, which can be considered as archetypes of the bright discs we modelled here. We also note that both of these discs were previously best fit by assuming two-belt models, mainly to account for the blue colour and the additional emission in the mid-IR, which our results suggest may just be an artefact of a single very bright debris discs (see Sect. 4.4). We also note that the bright debris discs for which a colour index has been derived include a clear non-blue case: HR4796, which has a red colour in the $\lambda \sim 0.5-1.6 \mu \mathrm{m}$ domain (Debes et al. 2008a). More scattered-light observations of very bright discs are thus needed to confirm this predicted correlation between fractional luminosity and blue colour. These blue discs may, however, be harder to detect (even though they have high fractional luminosities) on average because of their bluer colour, which lowers their fluxes at increasing wavelengths compared to a standard (redder) bright disc.

The effect of $s \leq s_{\text {blow }}$ grains is less easy to identify for the solar-type cases because regardless of whether $s \leq s_{\text {blow }}$ grains are included, a natural bump occurs in the spectrum around $\lambda \sim 1-1.5 \mu \mathrm{m}$ (see Fig. 15). This bump is due to the $s \sim s_{\text {blow }}=0.4 \mu \mathrm{m}$ grains that dominate the flux for a G2V star, which have a scattering coefficient that reaches a peak value close to $Q_{\text {sca }} \sim 4$ around $\lambda \sim 1 \mu \mathrm{m}$ (Fig. 15) ${ }^{6}$. As a consequence, the scattered-light spectrum of a disc around a solar-type star is naturally red in the $\lambda \sim 0.5-1 \mu \mathrm{m}$ domain and blue in the $\lambda \sim 1-2.5 \mu \mathrm{m}$ range. The presence of unbound grains is not able to reverse these two slopes, but it can significantly flatten them, especially for very bright warm asteroid-belt-like discs, as illustrated in the right panel of Fig. 14.

In this exploratory work, we have neglected the potential role of the scattering phase function (SPF) on disc colours, and have considered isotropic scattering for all grains. This aspect, as well as other problems that exceed the scope of this paper (see Sect. 5), will be explored in future studies. However, we expect that the SPF will not radically change our main conclusions on disc colours. Firstly, pronounced anisotropic effects occur only for discs that are viewed close to edge-on (Mulders et al. 2013), which only represent a fraction of possible viewing angles, and

\footnotetext{
6 This comes from the well-known result that grains of a given size are very efficient scatterers at wavelengths slightly larger than their size, for which they can scatter more electromagnetic energy flux than the flux that flows through their geometric cross section (Zubko 2013; Kennedy et al. 2015).
}

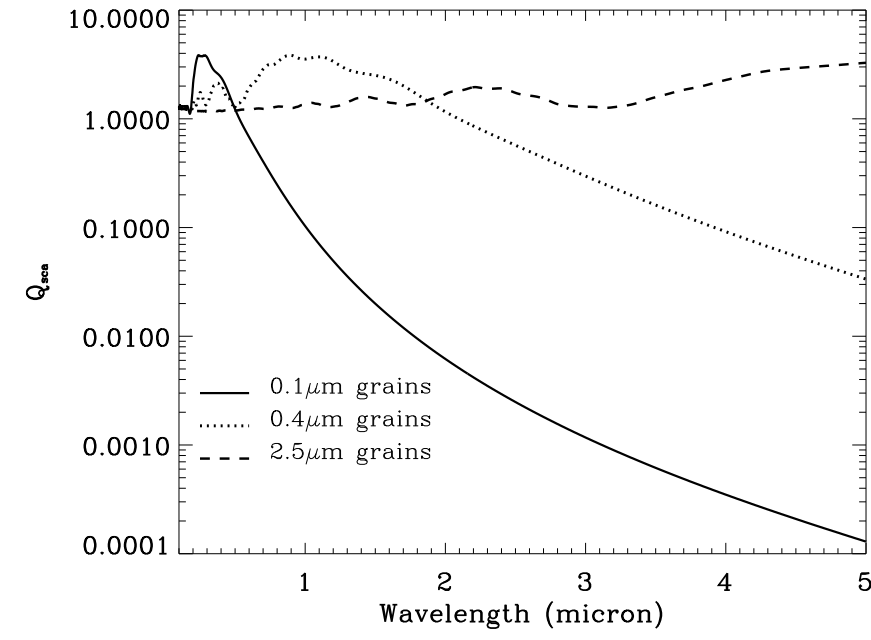

Fig. 15. $Q_{\text {sca }}(\lambda)$ values for three different compact silicate grain sizes.

it will occur only for the innermost projected separations. Secondly, the anisotropies of the SPF are expected to be maximum for grains much larger than $\lambda / 2 \pi$ (Mulders et al. 2013; Milli et al. 2017), whereas the colour effect we focus on here is mainly due to sub-micron grains that do not meet this criterion.

\subsection{Double belts?}

We find that bright discs around A stars naturally produce a substantial number of sub-micron grains that boost the signal in the $\sim 10-20 \mu \mathrm{m}$ wavelength region. Figure 16 shows that this excess mid-IR flux might mimic the emission from an additional warmer belt instead, and that our synthetic SED for the very bright disc case can be reasonably well fitted by the combination of two modified black-body curves, where the Rayleigh-Jeans part of the spectrum is corrected by a factor $\left(\lambda / \lambda_{0}\right)^{\gamma}$ for $\lambda \geq \lambda_{0}$, with $T=80 \mathrm{~K}$ and $T=115 \mathrm{~K}$, respectively. However, this effect might not be sufficient to explain most of the double belts, or more exactly, double-temperature configurations that have been inferred for very many debris discs (Kennedy \& Wyatt 2013; Geiler \& Krivov 2017). In most cases, the derived temperature ratio $R_{\mathrm{T}}$ between the two potential components of these systems is indeed of the order of a factor 2-4 (Kennedy \& Wyatt 2013), whereas even for our most extreme case, the temperature ratio 


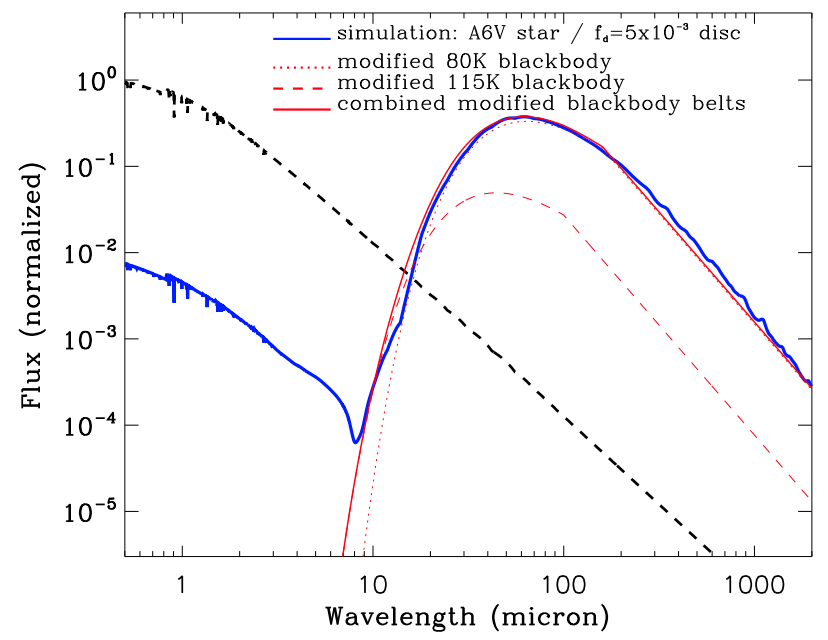

Fig. 16. Comparison between synthetic SED from simulation results (for an A6V star and a cold disc with $f_{\mathrm{d}}=5 \times 10^{-3}$ ) and a fit with the combination of two modified black-bodies with $T=115 \mathrm{~K}, \lambda_{0}=100 \mu \mathrm{m}$, $\gamma=-0.60$ and $T=80 \mathrm{~K}, \lambda_{0}=160 \mu \mathrm{m}, \gamma=-0.57$, respectively (see text for more detail).

between our two potential belts is limited to $\sim 1.5$ (corresponding to a separation of a factor $\sim(1.5)^{2}=2.25$ in radial distance). The temperature ratio between that of our smallest sub-micron grains and that of the largest black-body-like particles is higher than this, closer to a factor 2, but these larger grains only dominate the SED at wavelengths $\gtrsim 100 \mu \mathrm{m}$ and cannot shift the temperature of the cold component of our double-belt fit down to a black-body value. Kennedy \& Wyatt (2013) also explored the possibility that the range of observed temperatures could arise from different grain sizes in a single belt, but also ruled this scenario out on the same grounds: large black-body grains do not contribute enough to stretch the temperature range to observed values. However, this previous analysis did not take any contribution from $s \leq s_{\text {blow }}$ grains into account.

Our results could, however, be applied to the few unresolved (or poorly resolved) systems for which double temperatures with a ratio $R_{\mathrm{T}} \leq 2$ have been inferred. According to the two-grain model fit by Mittal et al. (2015), five systems potentially match this criterion: HD $15745\left(R_{\mathrm{T}}=1.79\right)$, HD $23267\left(R_{\mathrm{T}}=1.88\right)$, HD $108904\left(R_{\mathrm{T}}=1.56\right)$, HD $120326\left(R_{\mathrm{T}}=2.02\right)$, and HD 147010 $\left(R_{\mathrm{T}}=1.88\right)$.

\subsection{Importance of halos}

A peripheral but important result derived from our simulations is the important contribution that the halo, defined as all the matter that populates the region extending beyond the outer edge of the main belt, has to the total luminosity of the system. In contrast to the other results we highlighted in this work, this contribution is to a first order independent of the disc brightness or density (expressed by $f_{\mathrm{d}}$ ). This can be understood by the fact that the luminosity of this halo is for all explored cases dominated by small bound grains, and unbound particles only make a minor contribution. Because the number of these small bound grains is directly proportional to the density in the main disc (because they are collisionally produced and destroyed in this disc), their relative contribution compared to that of the main disc remains constant regardless of the disc density (for a more thorough discussion on the production and destruction of halo grains, see Strubbe \& Chiang 2006 and Thebault \& Wu 2008).
With the exception of a narrow dip at the transition from scattered light to thermal emission, we find that for all considered cases, the halo contributes to roughly $\sim 50 \%$ of the flux up to $\lambda \sim 50 \mu \mathrm{m}$ (see Figs. 4 and 7). Its contribution decreases at longer wavelengths because the small grains it is made of become poor emitters at wavelengths much longer than their size. These results have important consequences for observationally unresolved systems, for which we conclude that all photometric fits have to take the contribution of the halo into account.

\section{Conclusions and perspectives}

The observed characteristics of some specific debris discs point towards the presence of sub-micron grains, which seems counterintuitive given that such grains should be blown out by radiation pressure on short timescales. As a consequence, this presence of small unbound $s \leq s_{\text {blow }}$ dust is often interpreted as the result of some transient and/or violent event.

We revisited this question here by exploring to what extent the presence of these small grains could be explained by the natural collisional evolution of a bright debris disc with a high collisional activity. To this effect, we use a numerical code to estimate the fraction of $s \leq s_{\text {blow }}$ grains in bright debris discs at collisional steady state. We considered two different stellar types (A6V and G2V), two different disc types (warm asteroid-like and cold Kuiper belt-like), and two different disc brightnesses (a bright disc with a fractional luminosity $f_{\mathrm{d}}=10^{-3}$, and a very bright disc with $f_{\mathrm{d}}=5 \times 10^{-3}$ ).

For A stars, we find that small unbound grains always leave a detectable signature in bright discs with $f_{\mathrm{d}} \gtrsim 10^{-3}$. In addition to their own contribution to the disc luminosity, the grains are also able to efficiently erode the smallest bound grains with $s \geq s_{\text {blow }}$. The effect of unbound grains is most noticeable in two distinct locations of the disc SED: in scattered light in the optical to near-IR domain $(0.5 \lesssim \lambda \lesssim 3 \mu \mathrm{m})$, and in thermal emission in the $10 \lesssim \lambda \lesssim 20 \mu \mathrm{m}$ region. In scattered light, small unbound grains are able to change the disc colour from red to blue. In the mid-IR, the higher temperature of $s \leq s_{\text {blow }}$ grains compensates for their lower emissivity. This effect is strongest for cold discs where we are in the Wien domain of the Planck function. In the $10 \lesssim \lambda \lesssim$ $20 \mu \mathrm{m}$ range, sub-micron grains boost the disc luminosity by at least a factor of 2, which might allow some systems (especially those with cold discs) to become detectable at these wavelengths. In addition, the silicate solid-state feature at $\sim 10 \mu \mathrm{m}$ also becomes clearly detectable, but this time only in the case of warm discs. We also find that the additional luminosity contribution due to unbound grains can mimic the SED of two debris belts separated by a factor of $\sim 2$ in radial distance, although the effect might not be powerful enough to explain the majority of double-belt systems that have been inferred from observations, for which the expected separation between the belts is closer to a factor 5-10.

For G stars, $s \leq s_{\text {blow }}$ grains can account for more than $90 \%$ of the total geometrical cross section of the disc. However, their effect on the disc luminosity remains limited, mainly because the $s_{\text {blow }} \sim 0.4 \mu \mathrm{m}$ limit is itself in the sub-micron range, so that the $s \leq s_{\text {blow }}$ domain is confined to extremely small grains whose higher temperature can no longer mitigate their low emissivity. The $\sim 10 \mu \mathrm{m}$ silicate band is clearly visible, but it is mostly due to the smallest grains on bound orbits just above the $s_{\text {blow }}$ limit. For the disc colour in scattered light, the contribution of $s \leq s_{\text {blow }}$ grains is able to flatten the natural bell shape of the spectrum in the $0.5-2.5 \mu \mathrm{m}$ range, which is due to the peak in scattering 
efficiency around $\lambda \sim 1 \mu \mathrm{m}$ of the $s \sim s_{\text {blow }}$ grains that dominate the flux.

With the data at hand, it is too early to test all of our predictions, such as identifying a correlation between high fractional luminosity discs and blue colours, or the possibility of detecting cold bright discs with silicate features, because of small statistics issues. However, the James Webb Space Telescope (JWST) will be a powerful ally in the close future, both by potentially discovering more blue discs with the NIRCam instrument and by increasing the number of cold and warm discs with detected silicate features (with MIRI). We note that our results predict that bright discs around A or early-type stars will also be brighter than previously expected in the mid-IR (owing to the additional flux coming from the excess of sub-micron grains that are naturally produced in these discs), which will improve the odds of detecting debris discs in the mid-IR with the JWST.

There are of course some aspects that exceed the scope of the present exploratory study, whose main purpose was to identify a potentially important and so far neglected consequence of the collisional steady state in debris discs. These aspects, such as a thorough investigation of the role of grain physical composition, of the scattering phase function, or the potentially important role of the stellar magnetic field and stellar wind on sub-micron grains, are deferred to future work, which will also explore the specific cases of some individual debris discs.

Acknowledgements. The authors warmly thank Julien Milli, Grant Kennedy, and Jean-Charles Augereau for enlightening discussions.

\section{References}

Augereau, J. C., \& Beust, H. 2006, A\&A, 455, 987

Augereau, J. C., Lagrange, A. M., Mouillet, D., Papaloizou, J. C. B., \& Grorod, P.A. 1999, A\&A, 358, 557

Ballering, N. P., Rieke, G. H., Gáspár, A. 2014, ApJ, 793, 57

Beichman, C. A., Bryden, G., Gautier, T. N., et al. 2005, ApJ, 626, 1061

Benz, W., \& Asphaug, E. 1999, Icarus, 142, 5

Campo Bagatin, A., Cellino, A., Davis, D. R., Farinella, P., \& Paolicchi, P. 1994 P\&SS, 42, 1079

Chen, C. H., Mittal, T., Kuchner, M., et al. 2014, ApJS, 211, 25

Debes, J., Weinberger, A. J., Schneider, G. 2008a, ApJ, 673, 191

Debes, J., Weinberger, A. J., Song, I. 2008b, ApJ, 684, 41

Decin, G., Dominik, C., Malfait, K., Mayor, M., \& Waelkens, C. 2000, A\&A, 357,533

Draine, B. T. 2003, ARA\&A, 41, 241

Esposito, T. M., Fitzgerald, M. P., Grahams, J. R., et al., 2016, AJ, 152, 85

Fitzgerald, M. P., Kalas, P. G., \& Graham, J. R. 2007a, ApJ, 670, 557

Fitzgerald, M. P., Kalas, P. G., Duchene, G., Pinte, C., \& Graham, J. R. 2007b, ApJ, 670, 536

Flynn, G. J., \& Durda, D. D. 2004, P\&SS, 52, 1129

Gáspár, A., Psaltis, D., Rieke, G. H., \& Özel, F. 2012, ApJ, 754, 74

Geiler, F., \& Krivov, A. V. 2017, MNRAS, 468, 959

Grigorieva, A., Artymowicz, P., \& Thebault, P. 2007, A\&A, 461, 537
Heng, K., \& Tremaine, S. 2010, MNRAS, 401, 867 Henning, T. 2010, ARA\&A, 48, 21

Housen, K. R., \& Holsapple, K. A. 1999, Icarus, 142, 21 Johnson, B. C., Lisse, C. M., Chen, C. H., et al. 2012, ApJ, 761, 45 Jura, M., Malkan, M., White, R., et al. 1998, ApJ, 505, 897 Kalas, P. 2005, ApJ, 635, 169

Kalas, P., Fitzgerald, M. P., Graham, J. R. 2007, ApJ, 661, L85

Kennedy, G. M., \& Wyatt, M. C. 2013, MNRAS, 434, 3164

Kennedy, G. M., Matra, L., Marmier, M., et al., 2015, MNRAS, 449, 3121

Krijt, S., \& Kama, M. 2014, A\&A, 566, A2

Kral, Q., Thebault, P., \& Charnoz, S. 2013, A\&A, 558, A121

Kral, Q., Thebault, P., Augereau, J.-C., Boccaletti, A., \& Charnoz, S. 2015, A\&A, 573, A39

Kral, Q., Krivov, A. V., Defrere, D., et al. 2017, Astron. Rev., 13, 69

Krivov, A. V., Kimura, H., \& Mann, I. 1998, Icarus, 134, 311

Krivov, A. V., Mann, I., \& Krivova, N. A. 2000, A\&A, 362, 1127

Krivov, A. V., Löhne, T., \& Sremcević, M. 2006, A\&A, 455, 509

Krivov, A. V., Ide, A., Löhne, J. A., \& Blum, J. 2018, MNRAS, 474, 2564

Li, A. 2008, in Small Bodies in Planetary Sciences, Lecture Notes in Physics vol. 758, eds. I. Mann, A. Nakamura, \& T. Mukai (Heidelberg: Springer), 167

Lisse, C. M., Beichman, C. A., Bryden, G., \& Wyatt, M. C. 2007, ApJ, 658, 584

Lisse, C. M., Chen, C. H., Wyatt, M. C., \& Morlok, A. 2008, ApJ, 673, 1106

Lisse, C. M., Chen, C. H., Wyatt, M. C., et al. 2009, ApJ, 701, 2019

Lomax, J. R., Wisniewski, J. P., Roberge, et al. 2018, AJ, 155, 62

Matthews, B. C., Krivov, A. V., Wyatt, M. C., Bryden, G., \& Eiroa, C. 2014, in Protostars and Planets VI, eds. H. Beuther, R. S. Klessen, C. P. Dullemond, \& T. Henning (Tucson, AZ: University of Arizona Press), 521

Meyer, M. R., Backman, D. E., Weinberger, A. J., \& Wyatt, M. C. 2007, in Protostars and Planets V, eds. B. Reipurth, D. Jewitt, \& K. Keil (Tucson, AZ: University of Arizona Press), 573

Milli, J., Vigan, A., Mouillet, D., et al. 2017, A\&A, 599, A108

Mittal, T., Chen, C. H., Jang-Condell, H., et al. 2015, ApJ, 798, 97

Moor, A., Abraham, P., Derekas, A., et al. 2006, ApJ, 644, 525

Mulders, G. D., Min, M., Dominik, C., Debes, J. H., \& Schneider, G. 2013, A\&A, 549, A112

Nagaoka, H., Takasawa, S., Nakamura, A. M., \& Sangen, K. 2014, Meteorit. Planet. Sci., 49, 69

Olofsson, J., Augereau, J.-C., van Dishoeck, E. F., et al. 2010, A\&A, 520, A39

Olofsson, J., Henning, Th., Nielbock, M., et al. 2013, A\&A, 552, A4

Okamoto, Y. K., Kataza, H., Honda, M., et al. 2004, Nature, 431, 660

Pawellek, N., \& Krivov, A. 2015, MNRAS, 454, 3207

Pawellek, N., Krivov, A., Marshall, J. P., et al. 2014, ApJ, 792, 65

Stewart, S. T., \& Leinhardt, Z. 2009, ApJ, 691, 133

Strubbe, L. E., \& Chiang, E. I. 2006, ApJ, 648, 652

Telesco, C. M., Fisher, R. S., Wyatt, M. C., et al. 2005, Nature, 433, 133

Thebault, P. 2009, A\&A, 505, 1269

Thebault, P. 2016, A\&A, 587, A88

Thebault, P., \& Augereau, J. C. 2007, A\&A, 472, 169

Thebault, P., \& Kral, Q. 2018, A\&A, 609, A98

Thebault, P., \& Wu, Y. 2008, A\&A, 481, 713

Thebault, P., Augereau, J. C., \& Beust, H. 2003, A\&A, 408, 775

Thebault, P., Marzari, F., \& Scholl, H. 2006, Icarus, 183, 193

van Lieshout, R., Dominik, C., Kama, M., \& Min, M. 2014, A\&A, 571, A51

Voshchinnikov, N. V., \& Henning, T. 2008, A\&A, 483, 9

Weinberger, A. J., Becklin, E. E., Song, I., \& Zuckerman, B. 2011, ApJ, 726, 72

Wyatt, M. C. 2008, ARA\&A, 46, 339

Zubko, E. 2013, Earth Planet Space, 65, 139

Zuckerman, B., \& Song, I. 2012, ApJ, 758, 77 